\title{
Estudo de Caso de Controle Supervisório Tolerante a Perdas Intermitentes de Observação: Planta MECATRIME*
}

\author{
Rárisson Queiroz Hilário* \\ Antonio Eduardo Carrilho da Cunha* \\ * Instituto Militar de Engenharia - IME \\ Programa de Pós-Graduação em Engenharia Elétrica \\ Praça General Tibúrcio, 80 \\ Rio de Janeiro, RJ, Brasil \\ CEP: 22290-270 (e-mail: rarimhilario@gmail.com, \\ carrilho@ime.eb.br).
}

\begin{abstract}
This paper presents an application for robust control problem based on a CIM system which belongs to Military Engineering Institute, called MECATRIME. A case study was conducted with the objective of treating the intermittent loss of event observations due to a communication failure. Initially, been developed a mapping of the system done to identify the points or situations in which it was possible loss observation based on loss communication and after this mapping, a system modeling was performed in automata with an observation intermittent. A synthesis of the supervisor was also presented and presented as a formal tool to be used to guide an achievement of the system design.

Resumo: Neste artigo é apresentada uma aplicação ao problema de controle supervisório robusto baseado em um sistema CIM existente no Instituto Militar de Engenharia, chamado de MECATRIME. Fez-se um estudo de caso com o objetivo de tratar problemas de perdas intermitentes de observação devido a falhas de comunicação. Inicialmente, foi feito um mapeamento do sistema para identificar os pontos ou situações em que há possibilidade de perda de observação em função de falha de comunicação e após esse mapeamento foi realizada a modelagem do sistema baseado em autômatos com a perda intermitente de observação. Foi feita também a síntese do supervisor e foi mostrado como uma ferramenta formal pode ser utilizada para guiar a realização do projeto do sistema.
\end{abstract}

Keywords: Discrete Event Systems, Supervisory Control, Intermittent Observation Losses, Manufacturing System, System Modeling.

Palavras-chaves: Sistemas a Eventos Discretos, Controle Supervisório, Perda Intermitente de Observação, Sistema de Manufatura, Modelagem de Sistema.

\section{INTRODUÇÃO}

Para Sistemas a Eventos Discretos (SEDs), a Teoria de Controle Supervisório (TCS) introduzida em Ramadge and Wonham (1989) define formalmente a síntese de controladores, permitindo que eles sejam seguros e ótimos. Originalmente, a abordagem sugere que a ocorrência de todos os eventos é observada, ou seja, propõe a ideia de que há um sensor que gera uma informação do acontecimento de um determinado evento.

Quando o supervisor não enxerga o acontecimento de alguns eventos, surge então o conceito de observação parcial. Esse conceito é abordado em Wonham and Cai (2017), sendo que a hipótese é a não existência de alguns sensores e portanto, para esses eventos, supõe-se que eles sejam não observáveis.

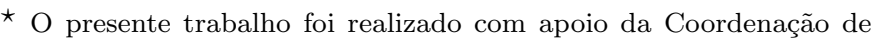
Aperfeiçoamento de Pessoal de Nível Superior - Brasil (CAPES) Código de Financiamento 001.
}

Em contrapartida, quando um evento é observável e o supervisor por alguma eventualidade não o enxerga, surge então o conceito de perdas intermitentes de observação que, diferentemente da observação parcial, há um sensor que monitora a ocorrência de um evento, mas por algum motivo, a observação deste evento não pôde ser registrada. Uma falha no sensor ou na comunicação pode causar a perda intermitente de observação (Carvalho et al., 2012).

Quando há sistemas em rede, faz-se necessário que haja garantia de segurança ponta a ponta, para que a informação da ocorrência de um evento seja transmitida. Esse problema de controle é tratado pelo controle supervisório sob falhas intermitentes de observação. Em Rohloff (2005), Sánchez and Montoya (2006) e Rohloff (2012), foi considerado o problema de controle supervisório seguro mediante a falha em um sistema, em que se supôs que poderia haver uma falha a qualquer instante e que, após sua ocorrência, a falha seria permanente. O trabalho feito em Alves (2014) aborda de maneira diferente o problema, 
permitindo a implantação de um supervisor que continue atuando mesmo que um evento se torne não observável de forma intermitente. Em Lin (2014), foi investigado o controle de SEDs em rede, em que foi considerado o atraso na comunicação e as perdas de observação.

No Controle Tolerante a Falhas (CTF), um dos objetivos ao sintetizar um supervisor que atenda um conjunto de especificações é garantir que ele atinja seu objetivo ou que pelo menos funcione de maneira segura, evitando que a planta seja interrompida devido a ocorrência de alguma falha, podendo o CTF ser abordado como ativo ou passivo (Paoli et al., 2011). Na abordagem ativa, com o diagnóstico ou prognóstico de uma falha, a lei de controle é adaptada ao comportamento do sistema quando este é cometido por algum tipo de falha, com o intuito de garantir que o objetivo do controle seja alcançado. Já na abordagem passiva, tem-se como objetivo encontrar um único controlador que atenda as especificações de controle tanto na operação nominal quanto após a ocorrência de falhas. Dentro dessas perspectivas, este trabalho se encaixa na abordagem passiva.

Este artigo é estruturado da seguinte forma: a seção 2 apresenta uma breve revisão da teoria de SEDs e revê o conceito para síntese de um supervisor sujeitos a perdas intermitentes de observação; na seção 3 é apresentada a planta MECATRIME, com sua composição, bem como o seu funcionamento e seus componentes; na seção 4 é apresentada a construção do nível hierárquico, abordando um contexto físico e lógico de uma das estações de trabalho da planta MECATRIME; na seção 5 são feitas considerações com o objetivo de realizar a síntese de um supervisor robusto quando houver falha de comunicação entre os módulos de uma estação; e na seção 6 são feitos os comentários finais e sugestões para prosseguimento deste trabalho.

\section{REVISÃO DA TEORIA DE CONTROLE SUPERVISÓRIO}

\subsection{Sistema a Eventos Discretos}

Nesta seção é feito um breve resumo de SEDs, tendo como base, o trabalho feito por Cassandras and Lafortune (2008). Em controle supervisório, o comportamento da planta é modelado por um autômato $G=$ $\left(X, \Sigma, f, x_{0}, X_{m}\right)$, sendo $X$ o conjunto de estados, $\Sigma$ o conjunto finito de eventos, $f: X \times \Sigma \rightarrow X$ é a função de transição, $x_{0}$ é o estado inicial e $X_{m}$ é o conjunto de estados marcados. As linguagens geradas e marcadas por um autômato $G$ são representadas respectivamente por $L(G)$, que representa todas as sequências de eventos que podem ocorrer a partir do estado inicial; e $L_{m}(G)$, que representa todas as sequências de eventos que terminam em um estado marcado (tarefa completa).

O conjunto de eventos $\Sigma$ pode ser particionado em $\Sigma=$ $\Sigma_{c} \dot{\cup} \Sigma_{u c}$, sendo que $\Sigma_{c}$ representa o conjunto de eventos controláveis (eventos que podem ser desabilitados por um supervisor) e $\Sigma_{u c}$ representa o conjunto de eventos não controláveis.

Outra forma de particionar o conjunto de eventos é $\Sigma=$ $\Sigma_{o} \dot{\cup} \Sigma_{u o}$, sendo que $\Sigma_{o}$ representa o conjunto de eventos observáveis e $\Sigma_{u o}$ representa o conjunto de eventos não observáveis. Os eventos não observáveis são classificados dessa forma quando no sistema não há sensores que monitoram suas ocorrências.

Uma operação importante envolvendo autômatos é a projeção natural ou simplesmente projeção. Em Ramadge and Wonham (1989), a projeção é definida no domínio $P_{o}: \Sigma^{*} \rightarrow \Sigma_{o}^{*}$. A projeção substitui os rótulos da transição de um autômato em $\Sigma^{*} \backslash \Sigma_{o}^{*}$ por $\varepsilon$, ou seja, os eventos substituídos são aqueles contidos em $\Sigma^{*}$ e não em $\Sigma_{o}^{*}$.

\subsection{Teoria de Controle Supervisório Sob Perda Intermitente de Observação}

O conjunto de eventos $\Sigma$ sofre uma nova partição, $\Sigma=$ $\Sigma_{\text {ilo }} \cup \Sigma_{\text {nilo }}$, em que $\Sigma_{\text {ilo }}$ representa o conjuntos de eventos passíveis de perdas intermitentes de observação, enquanto que $\Sigma_{\text {nilo }}$ representa o conjunto de eventos sempre observáveis. Ao considerar a perda intermitente de observação de um evento, define-se um novo conjunto de eventos não observáveis $\Sigma_{i l o}^{\prime}=\left\{\sigma^{\prime}: \sigma \in \Sigma_{i l o}\right\}$, sendo $\Sigma_{i l o}^{\prime}$ um conjunto de eventos fictício, construído a partir de $\Sigma_{i l o}$, em que cada evento $\sigma^{\prime} \in \Sigma_{i l o}^{\prime}$ possui correspondência biunívoca com o evento $\sigma \in \Sigma_{i l o}$. No trabalho de Carvalho et al. (2012) foi definida a operação de dilatação $D$, cujo mapeamento pode ser visto a seguir, definindo também um conjunto dilatado de eventos $\Sigma_{d i l}=\Sigma \dot{\cup} \Sigma_{i l o}^{\prime}$ :

$$
\begin{aligned}
D: & \Sigma^{*} \rightarrow 2^{\Sigma_{d i l}^{*}} \\
& s \mapsto D(s)
\end{aligned}
$$

sendo que

$$
\begin{aligned}
& D(\varepsilon)=\{\varepsilon\} \\
& D(\sigma)=\left\{\begin{array}{l}
\{\sigma\}, \quad \text { se } \sigma \in \Sigma \backslash \Sigma_{i l o} \\
\left\{\sigma, \sigma^{\prime}\right\}, \quad \text { se } \sigma \in \Sigma_{i l o}
\end{array}\right. \\
& D(s \sigma)=D(s) D(\sigma), \text { se } s \in \Sigma^{*}, \sigma \in \Sigma
\end{aligned}
$$

A extensão do mapeamento $D$ para uma linguagem $L \subseteq$ $\Sigma^{*}$ é dada por $D(L)=\bigcup_{s \in L} D(s)$.

Uma operação importante que envolve a dilatação é a compressão (Alves et al., 2019), que recupera uma palavra $s$ de qualquer palavra em $D(s)$. O mapeamento da operação de compressão pode ser visto a seguir:

$$
\begin{aligned}
C: \Sigma_{d i l}^{*} & \rightarrow \Sigma^{*} \\
s_{d i l} & \mapsto C\left(s_{d i l}\right)
\end{aligned}
$$

sendo que,

(i) $C(\varepsilon)=\varepsilon$

(ii) $C(\sigma)=\sigma$, se $\sigma \in \Sigma$

(iii) $C\left(\sigma^{\prime}\right)=\sigma$, se $\sigma^{\prime} \in \Sigma_{i l o}^{\prime}$

(vi) $C\left(s_{d i l} \sigma\right)=C\left(s_{d i l}\right) C(\sigma), \forall s_{d i l} \in \Sigma_{d i l}^{*}$ e $\sigma \in \Sigma_{d i l}$

A diferença entre as operações de projeção e compressão se dá pelo fato de $P_{d i l}$ suprimir eventos não observáveis presentes em qualquer palavra em $D(s)$, enquanto que a compressão recupera qualquer palavra em $D(s)$. Para exemplificar, considere $s_{d i l}=a b c^{\prime} b c^{\prime}$, então $P_{d i l}\left(s_{d i l}\right)=$ $a b b$ e $C\left(s_{d i l}\right)=a b c b c$.

\subsection{Supervisor Robusto}

$\mathrm{Na}$ Teoria de Controle Supervisório considerando que $\Sigma=$ $\Sigma_{o}$, tem-se que a interação entre supervisor $(S)$ e planta 


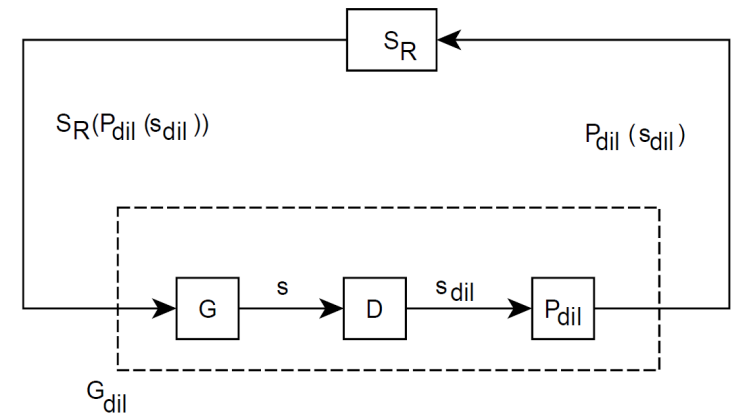

Figura 1. Representação de $S_{R}$ atuando sob $G_{d i l}$.

$(G)$ é feita de forma direta, ou seja, $S$ controla $G$ baseado na própria planta. Ao considerar que $\Sigma=\Sigma_{i l o} \cup \dot{U} \Sigma_{\text {nilo }}$, a forma como o supervisor (denominado agora como supervisor robusto $-S_{R}$ ) interage com $G$ é modificada pois, utilizando a abordagem de perda intermitente de observação, o supervisor agora atua sob a planta dilatada $G_{d i l}$ e não mais em $G$, seguida de $P_{d i l}$ (Alves et al., 2014). A Figura 1 mostra a representação de $S_{R}$ atuando sob $G_{d i l}$.

O supervisor $S_{R}$ é definidor por

$$
S_{R}: P_{d i l}\left(D\left(L\left(G_{d i l}\right)\right)\right) \rightarrow 2^{\Sigma_{d i l}}
$$

em que $P_{d i l}: \Sigma_{d i l}^{*} \rightarrow \Sigma_{o}^{*}$. A linguagem gerada por $S_{R}$ controlando $G_{d i l}, L\left(S_{R} / G_{d i l}\right)$, é dada como

1. $\varepsilon \in L\left(S_{R} / G_{d i l}\right)$; e

2. $\left(\forall s_{d i l} \in \Sigma_{d i l}^{*}\right)\left(\forall \sigma_{d i l} \in \Sigma_{d i l}\right), s_{d i l} \sigma_{d i l} \in L\left(S_{R} / G_{d i l}\right) \Leftrightarrow$ $\left(s_{d i l} \sigma_{d i l} \in L\left(G_{d i l}\right)\right) \wedge\left(s_{d i l} \in L\left(S_{R} / G_{d i l}\right)\right) \wedge\left(\sigma_{d i l} \in\right.$ $\left.S_{R}\left(P_{\text {dil }}\left(s_{\text {dil }}\right)\right)\right)$.

A linguagem gerada por $S_{R}$ controlando $G, L\left(S_{R} / G\right)$, é dada como $L\left(S_{R} / G\right)=C\left(L\left(S_{R} / G_{d i l}\right)\right)$.

\section{Problema de Controle Supervisório - Comporta- mento Dilatado}

Dado $G_{d i l}$ com $\Sigma_{d i l}=\Sigma_{d i l, c} \dot{\cup} \Sigma_{d i l, u c}, \Sigma=\Sigma_{o} \dot{U} \Sigma_{u o} e$ $D(K) \subseteq L\left(G_{d i l}\right)$, encontrar $S_{R}$ para $G_{d i l}$, tal que:

$$
\emptyset \neq L\left(S_{R} / G_{d i l}\right) \subseteq D(K)
$$

\section{Problema de Controle Supervisório - Comporta- mento Real}

Dado $G \operatorname{com} \Sigma=\Sigma_{c} \dot{\cup} \Sigma_{u c}, \Sigma=\Sigma_{\text {nilo }} \dot{U} \Sigma_{\text {ilo }} e K \subseteq L(G)$, encontrar $S_{R}$ para $G$, tal que:

$$
\emptyset \neq L\left(S_{R} / G\right)=K
$$

Pela definição dos supervisores que foi dada, os problemas de controle supervisório envolvendo o comportamento dilatado e o comportamento real são equivalentes. A seguir, são apresentadas duas importantes definições: controlabilidade e observabilidade.

Definição 1. (Controlabilidade) Sejam $L, K \subseteq \Sigma^{*}$, como $L=\bar{L}$ e $\Sigma_{d i l, u c} \subseteq \Sigma$. A linguagem $K \subseteq \Sigma^{*}$ é dita ser controlável em relação a $L\left(G_{d i l}\right)$ e $\Sigma_{d i l, u c}$ se $\bar{K} \Sigma_{u c} \cap L \subseteq \bar{K}$. Definição 2. (Observabilidade) Dada uma planta $G$ e uma projeção $P_{d i l}$, a linguagem $K \subseteq \Sigma^{*}$ é dita ser observável em relação a $L\left(G_{d i l}\right), \Sigma_{o}$ e $P_{d i l}$ se $\forall s, t \in \bar{K}, \sigma \in \Sigma$ : $P_{d i l}(s)=P_{d i l}(t), s \sigma \in \bar{K}, t \sigma \in L\left(G_{d i l}\right) \Rightarrow t \sigma \in \bar{K}$.

A partir da Definição 1 e da Definição 2, apresenta-se o teorema para uma linguagem controlável e observável.
Esse teorema foi adaptado de Alves et al. (2014) em cima dos conceitos que foram apresentados.

Teorema 3. Seja $G_{d i l} \operatorname{com} \Sigma_{d i l}=\Sigma_{d i l, c} \cup \dot{\cup} \Sigma_{d i l, u c}$ e $\Sigma=$ $\Sigma_{\text {nilo }} \dot{\cup} \Sigma_{i l o} \dot{\cup} \Sigma_{\text {ilo }}^{\prime}$ e $K \subseteq \bar{K} \subseteq D(K)$, então existe um supervisor $S_{R}$ para $G_{d i l}$, tal que $L\left(S_{R} / G_{d i l}\right)=K_{d i l}$ se, e somente se, $K_{d i l}$ for controlável (em relação a $L\left(G_{d i l}\right)$ e $\Sigma_{d i l, u c}$ ) e observável (em relação a $L\left(G_{d i l}\right), \Sigma_{o}$ e $\left.P_{d i l}\right)$.

O conjunto de ventos observáveis é dado como sendo $\Sigma_{o}=\Sigma_{\text {nilo }} \cup \dot{\cup} \Sigma_{i l o}$ e o conjunto de eventos não observáveis é dado como sendo $\Sigma_{u o}=\Sigma_{i l o}^{\prime}$.

A observabilidade possui como propriedade o fato de não ser fechada para a união, portanto não é possível encontrar uma suprema sublinguagem observável (SupO). Já a controlabilidade é fechada para a união e pode-se encontrar uma suprema sublinguagem controlável $($ SupC). Para o Problema de Controle Supervisório do comportamento dilatado, faz-se necessário encontrar uma linguagem que seja controlável e observável. Utiliza-se então o conceito de normalidade, que é fechada para união, permitindo encontrar uma suprema sublinguagem normal (SupN), e tal que, implica em observabilidade. Tendo $\Sigma_{c} \subseteq \Sigma_{o}, K$ controlável (em relação a $L\left(G_{\text {dil }}\right)$ e $\Sigma_{\text {diluc }}$ ) e $K$ observável (em relação a $L\left(G_{d i l}\right), \Sigma_{o}$ e $P_{d i l}$ ), então $K$ é normal (em relação a $L\left(G_{d i l}\right)$ e $\left.P_{d i l, u c}\right)$ e isso significa que uma suprema sublinguagem controlável e normal (SupCN) também é uma suprema sublinguagem controlável e observável (SupCO) (Cassandras and Lafortune, 2008).

Tendo isso em vista, segue a definição de Normalidade.

Definição 4. (Normalidade) Considerando $L=\bar{L} \subseteq \Sigma^{*} \mathrm{e}$ a projeção $P: \Sigma^{*} \rightarrow \Sigma_{o}^{*}$, a linguagem $K \subseteq L$ é dita ser normal em relação a $L\left(G_{d i l}\right)$ e $P_{d i l, u c}$ se

$$
\bar{K}=P^{-1}[P[\bar{K}]] \cap L .
$$

Por fim, optou-se pela solução de normalidade, sendo que existe solução por suprema linguagem relativa observável (SupRO) (Cai and Wonham, 2015) e por ínfimas superlinguagens $(\inf (K))$ (Alves, 2017), mas que não foram tratadas neste trabalho.

Existe solução para o problema de controle supervisório com comportamento real e para o problema de controle supervisório com comportamento dilatado se $\operatorname{Sup} C N(D(K))$ $\neq \emptyset$, controlável em relação a $L\left(G_{d i l}\right)$ e $\Sigma_{d i l, u c}$ e normal em relação a $L\left(G_{d i l}\right)$ e $P_{d i l}$. A solução para o comportamento dilatado e para o comportamento real pode ser vista a seguir.

\section{Solução do Comportamento Dilatado:}

$$
L\left(S_{R} / G_{d i l}\right)=\operatorname{SupCN}(D(K)) .
$$

\section{Solução do Comportamento Real:}

$$
L\left(S_{R} / G\right)=C(\operatorname{Sup} C N(D(K))) .
$$

\subsection{Passo a Passo}

Para realizar a modelagem do sistema MECATRIME, foram utilizadas abordagens com perspectiva física e lógica com o intuito de identificar os eventos passíveis de perda de observação e a partir disso, utilizar o seguinte passo a passo para se obter a síntese de um supervisor robusto: 


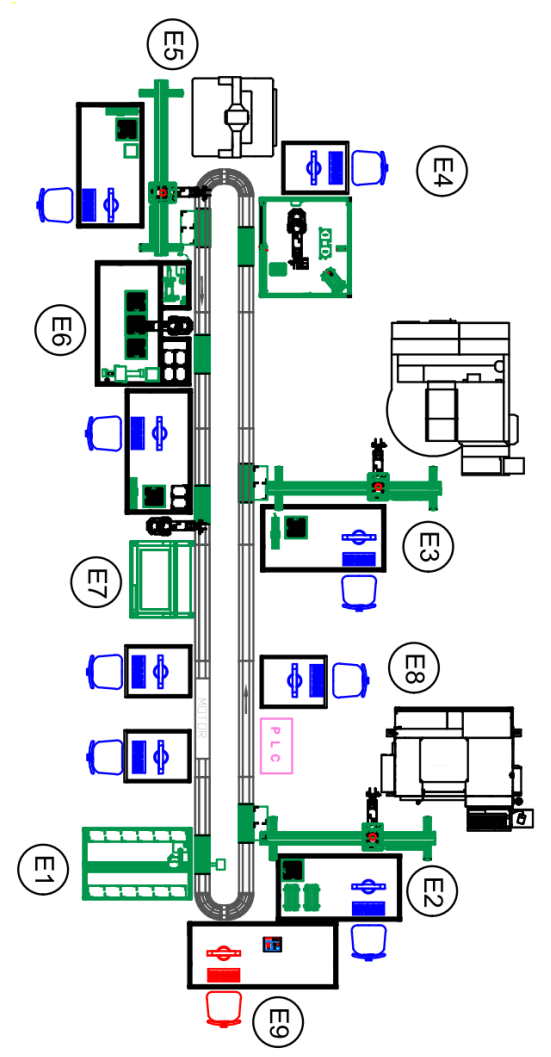

Figura 2. Desenho esquemático do sistema MECATRIME.

(1) Modelar a planta $G$ identificando $\Sigma_{c}, \Sigma_{u c}, \Sigma_{\text {nilo }}$ e $\Sigma_{i l o}$, $\operatorname{com} \Sigma_{u o}=\emptyset$

(2) Modelar as especificações como linguagem, prefixofechada, $E=\bar{E}$;

(3) Calcular a linguagem alvo $K=E \cap L(G)$, com $K \subseteq L(G)$ e $L(G)$ - fechada;

(4) Obter $G_{d i l}$, tal que $L\left(G_{d i l}\right)=D(L(G))$ e $L(G d i l)=$ $D(L(G))$, e $K_{d i l}=D(K)$

(5) Calcular $\operatorname{Sup} C N(D(K))$; e

(6) Se $\operatorname{Sup} C N(D(K))$ for não vazio, então o supervisor robusto é o reconhecedor de $P_{d i l}(\operatorname{Sup} C N(D(K)))$ e o comportamento em $M F$ será $C(\operatorname{Sup} C N(D(K)))$.

\section{SISTEMA MECATRIME}

A planta MECATRIME é classificada como um sistema CIM (Computer Integrated Manufacturing) e ela pode realizar a fabricação de peças e conjuntos de diversos perfis sendo mínima a intervenção humana. É composta por nove estações, sendo seis de produção, uma de controle da esteira, uma de gerenciamento e uma de armazenamento (ASRS). A Figura 2 mostra o desenho esquemático do sistema.

As estações de produção da planta MECATRIME são a Estação do Torno (E2), Estação da Fresa (E3), Estação de Solda (E4), Estação de Metrologia (CMM) (E5), Estação de Montagem e Inspeção Visual (E6) e Estação de Gravação a Laser (E7). As estações de Controle da Esteira, Gerenciamento (MANAGER) e Armazenagem (ASRS) são, respectivamente, $E 8, E 9$ e $E 1$.

A abordagem feita neste trabalho tem como base a produção local na estação do Torno, pois cada estação é equipada com matéria prima específica para a realização das tarefas referentes à cada estação sem que haja a necessidade de realizar as operações utilizando o ASRS e a esteira. Com as estações possuindo essa caracterítica, pode-se considerálas com Flexible Manufacturing System (FMS).

Para realizar as operações locais, todas estações, exceto $E 8$ e E9, possuem um software (SCORBASE) que por meio de um controlador (USB CONTROLLER), comanda as ações a serem realizadas. É o $S C O R B A S E$ que também realiza a integração de todos os componentes da estação e permite a atuação em alto nível do MANAGER. Nas estações $E 2$ a $E 7$ exite para cada uma delas um controlador denominado FS100 que é responsável por operar os movimentos dos robôs manipuladores presentes em cada uma dessas estações, além de permitir a atuação em alto nível do $S C O R B A S E$.

Na estação do MANAGER é executado o software Open$C I M$ que é responsável por coordenar todos os equipamentos do CIM, funcionando de forma simultânea tanto como sistema supervisório, quanto sistema para concepção e alteração do CIM, além de comandar as produções que devem ser feitas.

\section{IDENTIFICAÇÃO DE HIERARQUIAS - NÍVEL ESTAÇÃO}

Analisando a Estação do Torno, um subsistema da Planta MECATRIME, pôde-se construir os níveis hierárquicos de sistemas de automação dessa estação para a produção local de um produto (Sup_Brass - peça cilíndrica de latão torneada). No trabalho de Brandenbourger and Durand (2018) é possível observar que a divisão sugerida para os níveis hierárquicos considerando uma abordagem física foi feita da seguinte forma: Linha, Célula, Estação, Subestação, Módulo, Componente e Elemento. Especificamente para este trabalho, a hierarquia desenvolvida para a Estação do Torno é vista na Figura 3, sendo que as caixas de texto azuis representam eventos de comandos enviados para um componente do sistema ou então eventos de respostas recebidas pelos elementos do sistema, enquanto que as caixas de texto tracejadas representam o sinal lógico $(T A G)$ dos componentes e dos elementos físicos da estação do torno.

Após a construção da hierarquia física, realizou-se a hierarquização em um contexto lógico, que pode ser verificado na Figura 4, que mostra a interação que há entre o mundo cibernético com o mundo físico. Nela também se observa o fluxo de comunicação entre os diferentes níveis existentes na lógica de programação, em que o MANAGER se encontra um nível acima do USB Controller e do FS100. Tanto o USB Controller quanto o FS100 se comunicam com os componentes físicos que pertencem ao sistema. Observa-se com mais facilidade os comandos que são enviados para acionamento de componentes bem como o recebimento de uma informação por parte tanto do USB Controller quanto do $F S 100$. No interior das caixas de texto presentes nos blocos que representam o USB Controller e o FS100, estão os comandos das rotinas para os componentes que trafegam em meio físico, rede Ethernet e rede USB. Na Tabela 1 pode-se observar a relação entre alguns ventos e o tipo de comunicação em que estão inseridos. 
Tabela 1. Levantamento das Possíveis Causas para Perda de Observação de Eventos

\begin{tabular}{llll} 
Evento & Tipo de Comunicação & Possibilidade de Perda & Hipótese de Perda \\
\hline send_MSG & Ethernet & Comunicaçãa & Comunicação \\
end_Go_To_Position & USB & Comunicação ou Impacto Físico & Comunicação \\
I_LATHE_CYCLE_START & USB & Comunicação ou Impacto Físico & Comunicação \\
I_LATHE_CDOOR_CLOSED & USB & Comunicação ou Impacto Físico & Comunicação \\
end_JOB & Ethernet & Comunicação & Comunicação \\
\hline
\end{tabular}

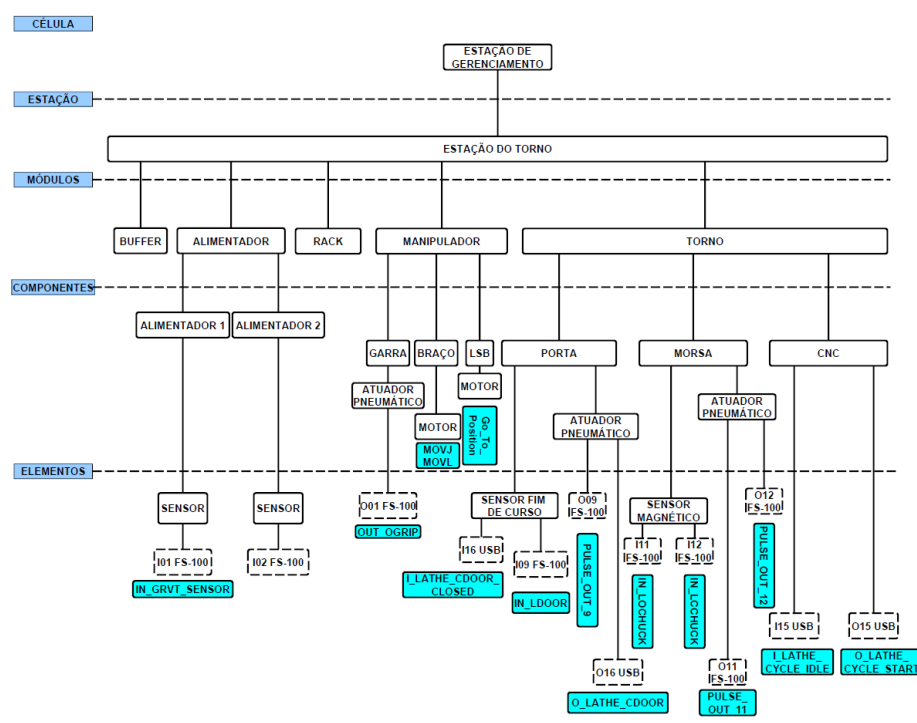

Figura 3. Abordagem física para o nível hierárquico da estação do Torno.

\section{RESULTADOS - PRODUÇÃO LOCAL}

\section{A. Modelagem dos Componentes ${ }^{1}$}

A construção dos autômatos foi baseada nas rotinas que o SCORBASE e o FS100 executam para a realização das tarefas, em que as linhas dos códigos se transformaram em eventos. Uma premissa de modelagem considerada foi de transformar cada linha que representa um comando com resposta em um evento de início (start) de tarefa ou em um evento de fim (end) de tarefa. Ainda pensando em eventos de início e fim de tarefas, serão considerados como controláveis os eventos de início e não controláveis os eventos de fim. No Script abaixo observa-se a rotina GET023 (SCORBASE).

\section{Set Subroutine GET023}

Call Subroutine SCRIPT.GET_FROM_GFRD1

Copy FS100 Position SCRIPT.P1 to Position 1001

Copy FS100 Position SCRIPT.P2 to Position 1002

Copy FS100 Position SCRIPT.P3 to Position 1003

Copy FS100 Position SCRIPT.P4 to Position 1004

Go to Position SCRIPT.PB1 Speed 50 (\%)

FS100 Start Job GT023

Send Message \$Start to MANAGER ID = TASK_ID

Return from Subroutine

$\mathrm{O}$ autômato que representa essa rotina é visto na Figura 5. A coloração vista na Figura 5 possui relação com as cores identificadas na Figura 4, sendo que a cor verde representa o MANAGER, a cor rosa o SCORBASE, a cor azul o FS100 e a cor laranja os componentes e elementos físicos

1 A modelagem foi feita considerando apenas os comportamentos gerados, sem marcação. presentes na estação do Torno. A disposição das cores indicam o sentido de comunicação. Por exemplo, o evento start_GET023 indica a comunicação entre MANAGER e $S C O R B A S E$, sendo que o comando é enviado pelo $M A$ NAGER e, consequentemente, recebido pelo SCORBASE. O sentido de comunicação é da esquerda para a direita. Há casos em que eventos não possuem nenhuma relação de interação, como por exemplo, o evento end_GET023, que indica apenas o fim de uma tarefa sem que haja a necessidade de aviso do fim dessa tarefa. Esse procedimento de construir autômatos foi feito para as demais rotinas do SCORBASE e FS100.

Para viabilizar a síntese, considerando a quantidade de componentes e redundâncias, criou-se uma simplificação em que foram retiradas as os eventos que indicam repetições. Para o SCORBASE por exemplo, start_copy_FS100_Position_Script.PX_GETO23 foi transformado em start_copy_FS100_Position_Script_GET023, sendo que o $X$ representa as posições que são copiadas. Também foram desconsiderados eventos em que não há compartilhamento de informação, por exemplo o evento end_GET023, em que a informação de fim dessa tarefa não é enviada para o MANAGER. O autômato simplificado da rotina GET023 pode ser visto na Figura 6.

Assim como foram criados autômatos representando o mundo cibernético da planta MECATRIME, também foram criados autômatos que representam o funcionamento físico do sistema. A Figura 7 mostra o autômato que representa o funcionamento da garra. Vale a pena ressaltar que os eventos que representam comando à planta foram considerados controláveis.

Para subseções $B$ e $C$, foram utilizados dois softwares para auxiliar nos cálculos do supervisor: o $I D E S^{2}$ 3 beta 1 e o UltraDES ${ }^{3}$. Na ferramenta IDES 3 beta 1 foi calculado os supervisores referentes à cada ação enviada pelo MANAGER ao SCORBASE. Já no UltraDES foi calculado o supervisor composto por todas as tarefas enviadas pelo MANAGER ao SCORBASE.

\section{B. Síntese Envolvendo Observação Completa}

Após a construção dos autômatos foi criada também uma especificação para atender o funcionamento adequado da produção do Sup_Brass. Essa especificação pode ser vista na Figura 8. A especificação foi criada com base na comunicação realizada entre MANAGER e SCORBASE.

A planta foi obtida realizando a composição entre os autômatos que representam o funcionamento físico do sistema, os autômatos que representam as rotinas do SCORBASE e FS100 e o MANAGER. A síntese do supervisor se deu calculando a suprema sublinguagem controlável $(\operatorname{Sup} C)$,

\footnotetext{
2 https://qshare.queensu.ca/Users01/rudie/www/software.html

3 http://lacsed.eng.ufmg.br/ultrades/
} 


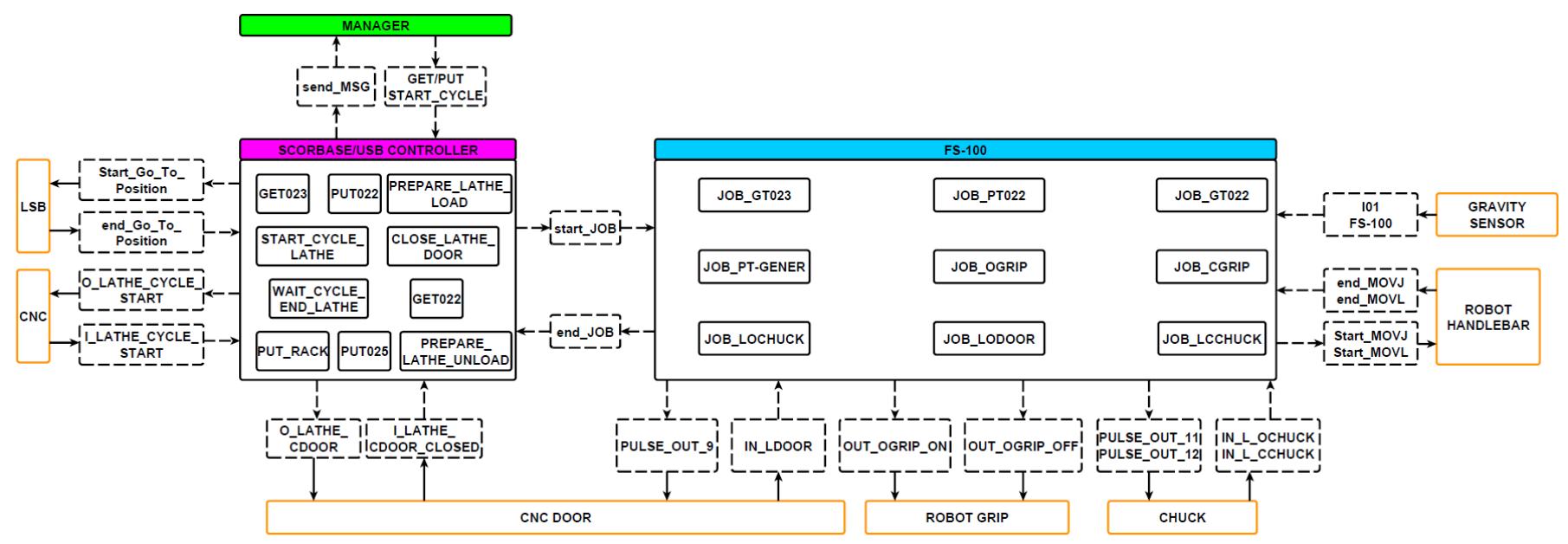

Figura 4. Abordagem lógica para o nível hierárquico da estação do Torno.

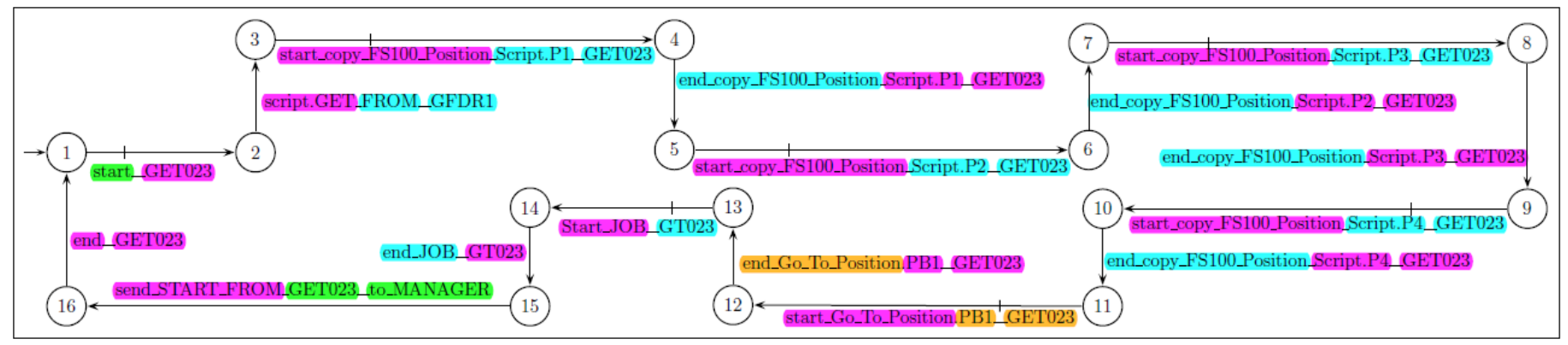

Figura 5. Autômato referente à rotina GET023 (SCORBASE).

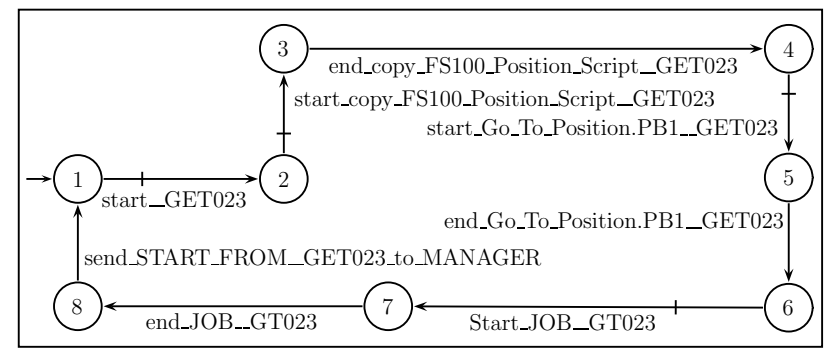

Figura 6. Autômato simplificado referente à rotina GET023.

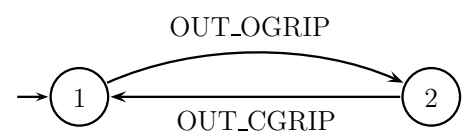

Figura 7. Autômato referente ao funcionamento físico da garra do robô.

obtendo um autômato com 127 estados. Foi possível verificar por inspeção que o supervisor atende aos requisitos de funcionamento da planta MECATRIME.

\section{Síntese Envolvendo Perda Intermitente de Observa- ção}

A partir da Figura 4, foram identificados os eventos que possuem chances de perdas de comunicação e alguns deles podem ser observar na Tabela 1 . O critério utilizado para definir se um evento é passível ou não de perda de observação é se o evento representa uma resposta à um comando por meio da Ethernet, como por exemplo, o evento send $\_M S G$ representa a reposta para o evento start_GET. Como primeira abordagem foi considerado que a perda de observação será devido à falha de comunicação entre $S C O R B A S E$ e FS100, ou seja, a resposta de tarefa realizada $(e n d J O B)$ não será recebida pelo $S C O R B A S E$. Também será considerado que os eventos que representam o mundo físico ou $U S B$ não serão passíveis de perdas de observação. A representação do evento passível de perda pode ser vista na Figura 9, que mostra o autômato dilatado referente à rotina GET023. Para os demais autômatos que possuem eventos que representam a resposta de tarefa realizada, também foram aplicados a dilatação desses eventos.

Após dilatar os eventos passíveis de perda de observação, foi realizada a síntese de um supervisor robusto, pelo cálculo da suprema sublinguagem controlável e normal $(S u p C N)$. Foi calculado o supervisor para cada tarefa que o MANAGER manda executar. O primeiro passo para a realização da síntese é aplicar a dilatação nos autômatos que representam a planta e a especificação do sistema e em seguida deve-se encontrar a linguagem alvo da planta. Feito isso, tenta-se encontrar a suprema sublinguagem controlável e normal para o sistema MECATRIME. Achada a suprema sublinguagem controlável e normal, calcula-se o observador dessa sublinguagem (para saber mais sobre observador, ver em Cassandras and Lafortune (2008)) e encontra-se um supervisor que atende aos requisitos de funcionamento do sistema. Observa-se uma incoerência na Figura 10, em que o supervisor encontrado referente a rotina GET023, permite que uma resposta de fim de tarefa seja enviada ao MANAGER mesmo que o FS100 


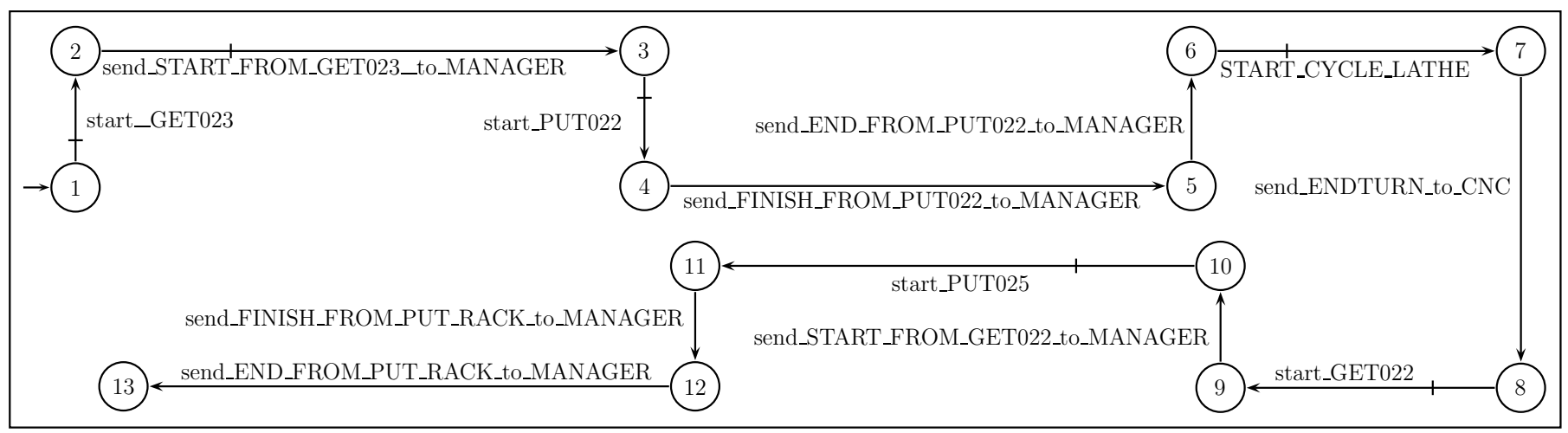

Figura 8. Especificação de funcionamento do sistema.

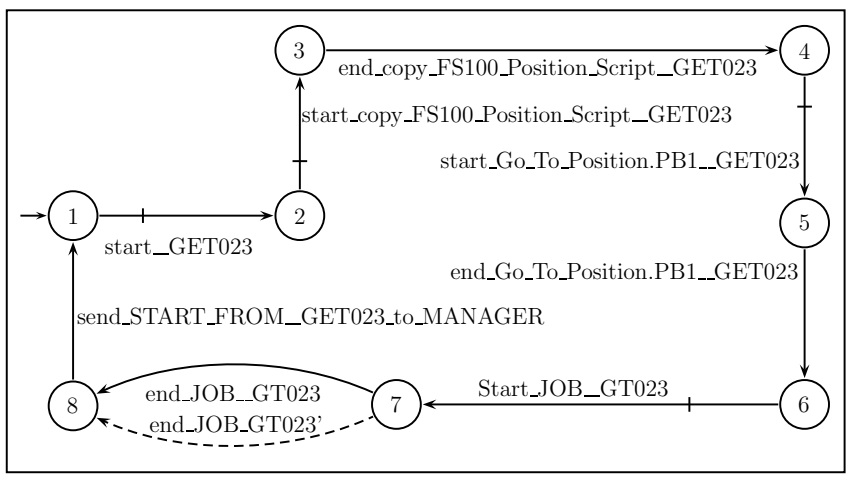

Figura 9. Autômato dilatado referente à rotina GET023.

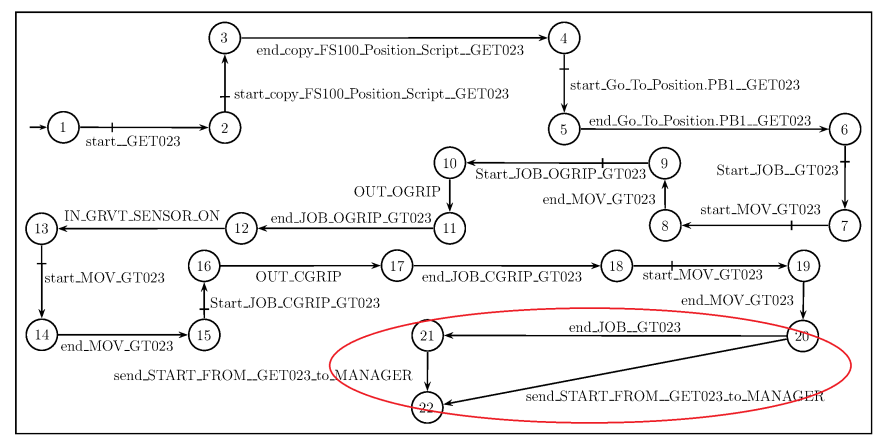

Figura 10. Supervisor apresentando incoerência.

não tenha indicado ao $S C O R B A S E$ que a realização da tarefa foi concluída.

Diante disso, pensou-se em uma nova abordagem com o intuito de evitar a ocorrência desse fato e chegou-se a um autômato que pode ser visto na Figura 11. Foi necessário também refinar o autômato referente a especificação, como visto na Figura 12. A construção desse autômato foi um refinamento feito no funcionamento do sistema, pois agora o MANAGER pode receber uma mensagem indicando a não realização de uma tarefa por falha de comunicação. A solução então é corrigir a programação atual, acrescentando uma linha de código que faça com que o $S C O R B A S E$ envie uma mensagem de erro para o $M A N A G E R$ toda vez que a comunicação entre $F S 100$ e $S C O R B A S E$ não for realizada.

Utilizando a abordagem da Figura 11, encontrou-se um supervisor que corrige a incoerência vista na Figura 10. O supervisor referente a rotina GET023 pode ser observado

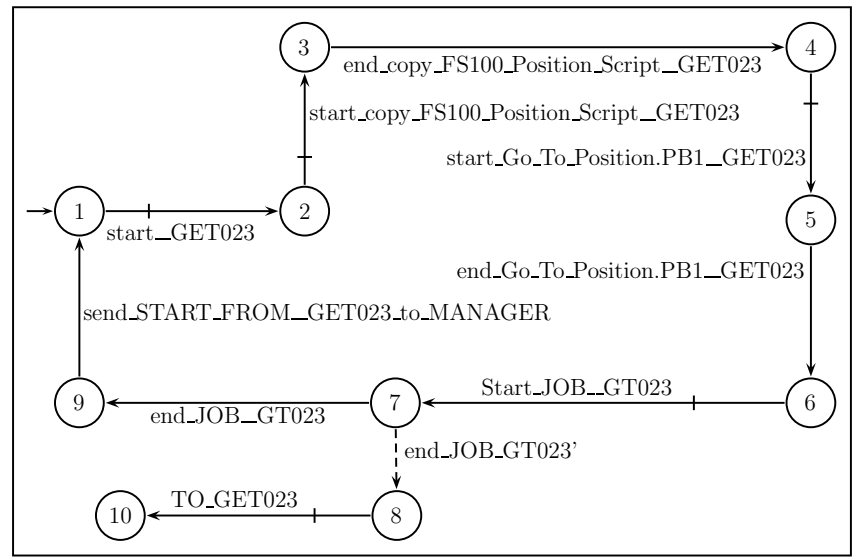

Figura 11. Autômato referente à rotina GET023 com nova abordagem de dilatação.

na Figura 13, que mostra a conclusão de uma tarefa apenas quando o supervisor enxerga a comunicação entre FS100 e $S C O R B A S E$

Foram obtidos também, supervisores referentes às demais rotinas que envolvem a produção local do torno. $\mathrm{O}$ supervisor geral encontrado possui 131 estados e foi possível verificar por inspeção que os requisitos de funcionamento da planta MECATRIME foram atendidos.

\section{CONCLUSÃO}

Neste artigo foi abordado o problema de controle supervisório robusto sob perdas intermitentes de observação, em que, com a modelagem realizada considerando o problema de síntese, foi possível aprimorar o modelo e consequentemente aprimorar a programação das rotinas da estação do torno da planta MECATRIME. Apesar de existirem outros cálculos para síntese de supervisor, como a suprema sublinguagem relativa e observável e ínfimas superlinguagens, apenas o cálculo da suprema sublinguagem controlável e normal foi abordado no escopo deste trabalho. Como continuidade deste trabalho, pretende-se realizar a modelagem do sistema abrangendo todas as estações e também devese utilizar a abordagem de observabilidade relativa, para assim, comparar qual abordagem é mais eficiente para o problema de perda de observação. 


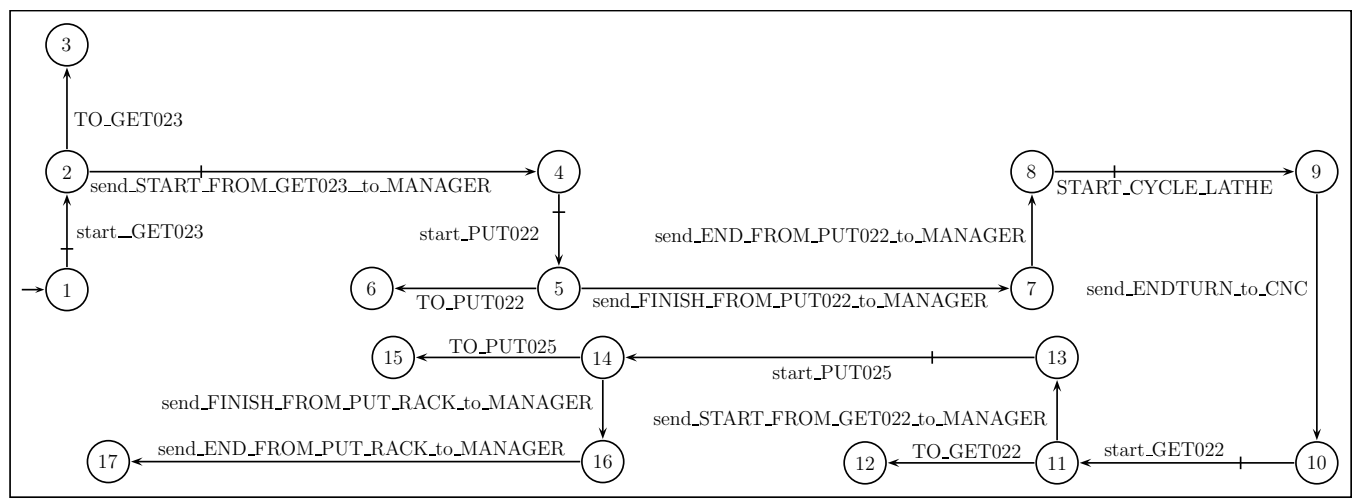

Figura 12. Especificação de funcionamento do sistema com nova abordagem.

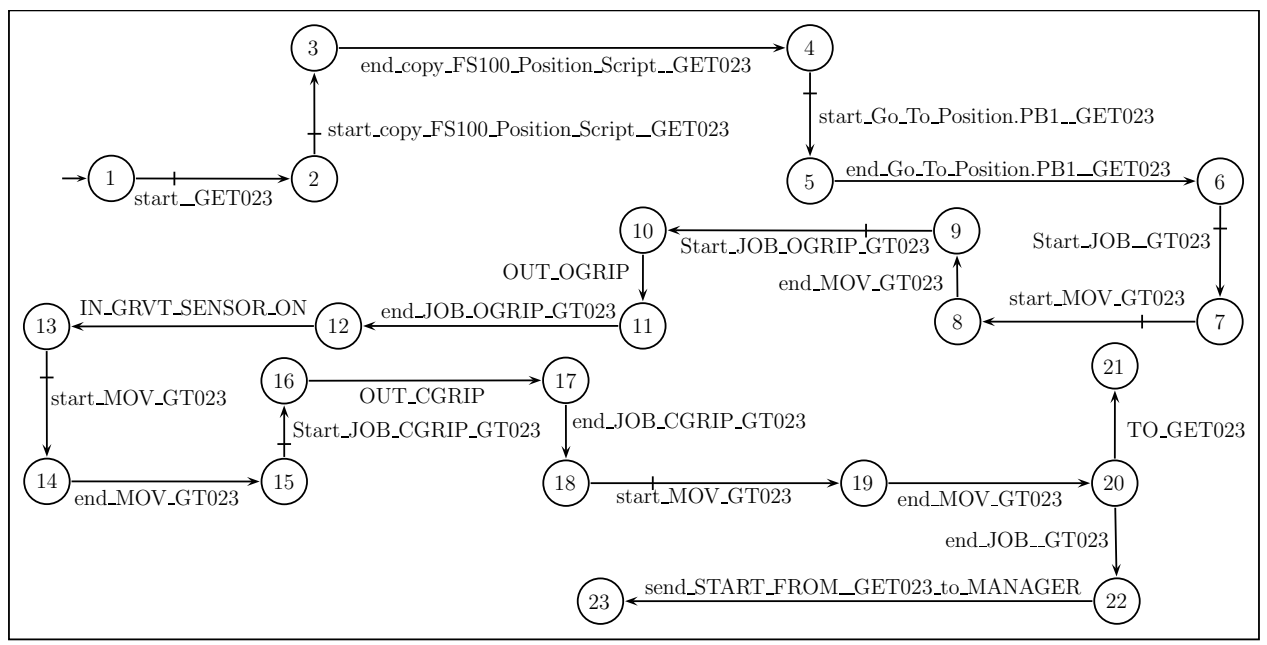

Figura 13. Supervisor utilizando nova abordagem.

\section{REFERÊNCIAS}

Alves, M.V.S., Basílio, J.C., da Cunha, A.E.C., Carvalho, L.K., and Moreira, M.V. (2014). Robust supervisory control against intermittent loss observations. In 12th IFAC/IEEE Workshop on Discrete Event Systems, 294 - 299. Cachan, France.

Alves, M.V.S., Cunha, A.E.C.D., Carvalho, L.K., Moreira, M.V., and Basilio, J.C. (2019). Robust supervisory control of discrete event systems against intermittent loss of observations. International Journal of Control.

Alves, M.V.S. (2014). Controle Supervisório Robusto de Sistemas a Eventos Discretos Sujeitos a Perdas Intermitentes de Observação. Master's thesis, Universidade Federal do Rio de Janeiro.

Alves, M.V.S. (2017). Supervisory Control of Networked Discrete Event Systems with Timing Structure. Ph.D. thesis, Universidade Federal do Rio de Janeiro.

Brandenbourger, B. and Durand, F. (2018). Design pattern for decomposition or aggregation of automation systems into hierarchy levels. In 2018 IEEE 23rd International Conference on Emerging Technologies and Factory Automation (ETFA), volume 1, 895-901. doi: 10.1109/ETFA.2018.8502627.

Cai, K. and Wonham, W.M. (2015). Relative observability of discrete-event systems and its supremal sublanguages. IEEE Transactions on Automatic Control, 60(3).

Carvalho, L.K., Moreira, M.V., and Basilio, J.C. (2012). Generalized robust diagnosability of discrete event sys- tems. Automatica, 48(9), 2068 - 2078.

Cassandras, C.G. and Lafortune, S. (2008). Introducion to Discrete Event Systems. Springer, $2^{\circ}$ edition.

Lin, F. (2014). Control of networked discrete eevent systems: Dealing with communication delays and losses. In SIAM Journal on Control and Optimization, volume 52, $1276-1298$.

Paoli, A., Sartini, M., and Lafortune, S. (2011). Active fault tolerant control of discrete event systems using online diagnostics. In Automatica, volume 47, 639-649.

Ramadge, P.J.G. and Wonham, W.M. (1989). The control of discrete event systems. Proceendings of the IEEE, 77, 81-98.

Rohloff, K.R. (2005). Failure tolerant supervisory control. In 44th IEEE Conference on Decision and Control, and the European Control Conference, 3493 - 3498. Seville, Spain.

Rohloff, K.R. (2012). Bounded sensor failure tolerant supervisory control. In 11th IFAC Workshop on Discrete Event Systems, volume 45, 272-277. Guadalajara, México.

Sánchez, A.M. and Montoya, F.J. (2006). Safe supervisory control under observability failure. Discrete Event Dynamic Systems, 16, 493-525.

Wonham, W.M. and Cai, K. (2017). Supervisory control of discrete-event systems. URL http://www. control.utoronto.ca/cgi-bin/dldes.cgi. Acessado em 20/05/2017. 